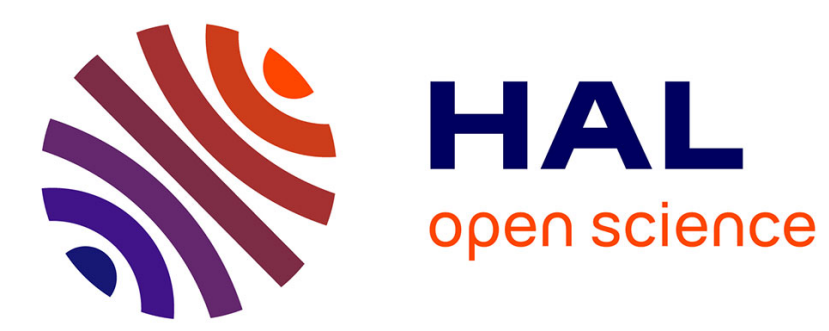

\title{
FILM. ONTOLOGIE DES IMAGES ET ICONOLOGIE AU-DELÀ DE L'HUMAIN
}

\author{
Jean-Michel Durafour
}

\section{To cite this version:}

Jean-Michel Durafour. FILM. ONTOLOGIE DES IMAGES ET ICONOLOGIE AU-DELÀ DE L'HUMAIN. Archives de Philosophie, 2018. hal-01868374v2

\section{HAL Id: hal-01868374 \\ https://hal.science/hal-01868374v2}

Submitted on 29 Dec 2020

HAL is a multi-disciplinary open access archive for the deposit and dissemination of scientific research documents, whether they are published or not. The documents may come from teaching and research institutions in France or abroad, or from public or private research centers.
L'archive ouverte pluridisciplinaire HAL, est destinée au dépôt et à la diffusion de documents scientifiques de niveau recherche, publiés ou non, émanant des établissements d'enseignement et de recherche français ou étrangers, des laboratoires publics ou privés. 
Film.

Ontologie des images et iconologie au-delà de l'humain

\author{
Jean-Michel DURAFOUR
}

\title{
paru initialement dans Archives de philosophie, tome 81, 2018/2, pp. 269-286 (version corrigée)
}

Le présent texte s'inscrit dans une réflexion philosophique sur l'iconologie et l'esthétique figurative ${ }^{1}$ exposée dans mon dernier ouvrage en date ${ }^{2}$. Cette pensée des images a pour nom éconologie ${ }^{3}$.

L'éconologie repose sur trois principes que je me permets de rappeler rapidement à l'ouverture de cet article (ils seront nécessaires pour situer exactement l'intervention philosophique qui sera la mienne dans quelques instants), et que je demande au lecteur de m'accorder.

1/ Les images en général sont des formes de vie. Il existe trois types de vie des images dans l'histoire de la théorie ou de l'histoire des images, artistiques mais pas seulement (scientifiques, politiques, etc.): imaginaire (mythe grec de Pygmalion, Portrait de Dorian Gray, Pinocchio), associative (la vie est réelle mais par métaphore ou analogie ${ }^{4}$ ), plénière. C'est en ce troisième sens que je propose de parler d'une vie des images : les images sont des formes de vie plénière non organique. Peu importe ici en quel sens précisément.

2/ En conséquence, les images entretiennent entre elles des relations mutualistes et de coévolution, d'entr'expression (spéculaire) mais aussi des relations matérielles d'entre-perception, c'est-à-dire que les images ne sont pas des choses mais des nœuds, des liaisons qui ne se limitent pas à la corrélation hommes-images. S'il ne nous est pas possible de connaître ces relations - puisque nous ne pouvons pas sortir de notre condition humaine - il nous est, en revanche, tout à fait loisible de les penser (d'en

\footnotetext{
${ }^{1}$ Je ne distingue pas ces deux termes comme on le fait usuellement. Le point n'est pas décisif ici.

${ }^{2}$ Cinéma et cristaux. Traité d'éconologie, Paris, Mimésis, coll. " Images, médiums », à paraître en mai 2018. Cet article en reprend les éléments portant sur le champ spéculatif ouvert par l'Ontologie orientée vers l'objet. Voir également L'Étrange Créature du lac noir de Jack Arnold. Aubades pour une zoologie des images, Aix-en-Provence, Rouge profond, coll. « Débords », 2017.

${ }^{3}$ Celle-ci se distingue complètement de ce que l'on trouve parfois sous le mot «éconologique » (par exemple, Alain Roger, Court Traité du paysage, Paris, Gallimard, coll. «Bibliothèque des Sciences humaines », 1997, p. 60) pour désigner des comportements au carrefour de l'écologie et de l'économie. ${ }^{4}$ Voir, par exemple, ce qu'écrit André Leroi-Gourhan à propos des techniques (qui sont des images) : «L'analyse des techniques montre que dans le temps elles se comportent à la manière des espèces vivantes, jouissant d'une forme d'évolution qui semble leur être propre et tendre à les faire échapper à l'emprise de l'homme » (Le Geste et la parole. Technique et langage [1964], Paris, Albin Michel, coll. «Sciences d'aujourd'hui, 1989, p. 206). La théorie des images en présente des variantes nombreuses, notamment pour ce qui est de l'époque contemporaine chez W. J. T. Mitchell (Que veulent les images? Une critique de la culture visuelle, traduit de l'anglais [États-Unis] par Maxime Boidy et Stéphane Roth, Dijon, Les Presses du réel, coll. «Perceptions », 2014), Horst Bredekamp (Théorie de l'acte d'image, traduit de l'allemand par Frédéric Joly en collaboration avec Yves Sintomer, Paris, La Découverte, coll. "Politique et sociétés», 2015) ou Carlo Severi (L'Objet-personne. Une anthropologie de la croyance visuelle, Paris, Éditions Rue d’Ulm/Musée du quai Branly, coll. « Asthetica », 2017).
} 
augmenter les intensités). L'éconologie est, à ce titre, une iconologie au-delà l'humain 5 .

3/ L'iconologie est une science des liens. Et pour rendre compte des nœuds vivants que sont les images, il faut acclimater aux images et à leur rapports des théories venues de la biologie (D'Arcy Thompson, Conrad H. Waddington), de l'écologie (Kinji Imanishi), mais aussi de la philosophie orientée vers l'objet (Timothy Morton), de l'anthropologie (Viveiros de Castro) ou des mathématiques (topologie, géométrie non euclidienne), et d'autres sciences encore selon les cas de figure. J'ai nommé nexialisme cette méthodologie elle-même relationniste et manipulant des interconcepts pour penser les images ${ }^{6}$.

Dans ce qui suit, il ne sera pas question directement de cinéma, mais des images en général. En revanche, les traits caractéristiques de l'éconologie sont pensés depuis les films. Je ne proposerai pas cette théorie éconologique si je n'étais d'abord un théoricien du cinéma. Je me permets ainsi de détourner légèrement la consigne de ce dossier. Je ne parlerai pas de cinéma et de philosophie, dans leurs rapports horizontaux ou d'entrelacement, mais je parlerai de l'un des moments philosophiques d'une science des images fondée à partir du cinéma: celui de la possibilité d'une iconologie et d'une esthétique au-delà de l'accès humain aux images, qui va prendre place dans le cadre plus large d'une iconologie orientée vers l'image théorisée à partir de certaines conceptions de l'Ontologie orientée vers l'objet développée depuis quelques années dans le monde anglo-saxon (Object-Oriented Ontology, $\mathrm{OOO}^{7}$ ), et dont on verra que, tout en ouvrant des perspectives tout à fait neuves sur les images, elles nous obligeront également à une grande vigilance méthodologique (notamment sur la question de leur mariage avec les théories relationnistes qui forment l'autre versant théorique de l'éconologie). Cet article se tient donc après le cinéma et après sa rencontre avec la philosophie dans la construction d'une autre théorie des images née à partir d'eux. Il est un exemple parmi d'autres de que le cinéma peut faire à la théorie esthétique ou iconologique des images.

Très précisément, pour en contextualiser les premiers soubresauts, les thèses qui seront développées dans un instant proviennent de réflexions antérieures sur la nature des figures filmiques en ce qu'elles n'apparaissent jamais à l'écran que par certaines de leurs faces. En travaillant sur la figure de l'homme-poisson de L'Étrange Créature $d u$ lac noir (1954) de Jack Arnold, j'ai été amené à proposer une approche des figures filmiques en objets non euclidiens n-dimensionnels dont chaque image ne donne que l'intersection sur une surface plane et illusoirement tridimensionnelle, c'est-à-dire dans un milieu (euclidien) adéquat à la perception humaine ${ }^{8}$.

La figure filmique n'existe jamais comme une totalité sommable à partir de ses différents aspects ponctuels (plans, points de vue, etc.) et s'enfonce à jamais derrière

\footnotetext{
${ }^{5}$ Je construis cette expression sur le modèle de l'anthropologie au-delà de l'humain théorisée - parmi d'autres (Viveiros de Castro, etc.) - par Eduardo Kohn: Comment pensent les forêts. Vers une anthropologie au-delà de l'humain [2013], traduit de l'anglais (États-Unis) par Grégory Delaplace, Bruxelles, Zones Sensibles, coll. « Pactum serva », 2016.

${ }^{6}$ Là encore, je n'invente pas le mot. Il se rencontre pour la première fois, sans aucune conceptualisation particulière, sous la plume de l'écrivain de science-fiction A. E. van Vogt à partir de la fin des années 1930 : cf. La Faune de l'espace, [1950], traduit de l'anglais (États-Unis) par Jean Rosenthal, Paris, J'ai Lu, 1979, p. 79 : « Le nexialisme est une science qui a pour but de coordonner les éléments d'un domaine de la connaissance avec ceux des autres domaines. »

${ }^{7}$ Le terme a été forgé par Graham Harman en 1999 (« Brief SR/OOO Tutorial», 23 juillet 2010, site Object-Oriented Philosophy, https://doctorzamalek2.wordpress.com/2010/07/23/brief-srooo-tutorial).

${ }^{8}$ L'Étrange Créature du lac noir de Jack Arnold. Aubades pour une zoologie des images, op. cit., p. 2024.
} 
les images, comme un calmar géant plongeant dans les abysses, en échappant en tant que telle à l'accès humain...

\section{VERS LA PHILOSOPHIE ORIENTEE VERS L'OBJET}

Avant de parler de l'Ontologie orientée vers l'objet, encore mal connue du lecteur français non anglophone, il faut sans doute commencer par dire deux mots du chemin - même très partiellement - qui y conduit pour comprendre d'où elle vient.

Toute la Critique de la raison pure repose sur une distinction centrale dans la philosophie kantienne, qui vient de plus loin (Dissertation de 1770), entre le phénomène, l'objet spatialisé et temporalisé tel qu'il apparaît à notre sensibilité, et la " chose en soi [Ding an sich $]^{9}$ ", c'est-à-dire l'objet tel qu'en lui-même à jamais inaperçu et inapercevable, dont le phénomène est la manifestation que nous pouvons seulement connaître. La pluie en soi n'est pas dans les gouttes, car celles-ci sont déjà des « objets empiriques ». Ce n'est jamais la pluie qui nous tombe sur la tête ou nous mouille : nous ne sommes trempés que par des «modifications ou éléments de notre intuition sensible ». L'objet ne se limite pas à ce que j'en perçois ni à ses qualités sensibles.

Il existe un inconnaissable de l'objet. Toute prétention d'un rapport de connaissance à des objets non phénoménalisables dans le cadre de l'expérience - âme, monde, réchauffement climatique, boson, radioactivité, bulle de Wolf-Rayet, capital, etc. mais aussi à la part non phénoménalisable de tout objet est vouée aux gémonies théoriques des illusions et des paralogismes. Mais cet inconnaissable n'est pas pour autant un impensable. Je peux le penser malgré tout, ne serait-ce que pour le penser comme inconnaissable ${ }^{10}$. Ce qui a pour nom ontologique «chose en soi », quand on se place du point de vue d'une réalité positivement intuitionnable sur un régime non sensible (et donc non humain), s'appelle "noumène » sous l'angle d'une théorie négative des limites de la science. Je peux penser la chose en soi, sinon je ne pourrai pas même dire qu'il existe des choses en soi, mais, en tant qu'être humain, je ne peux rien en connaître, puisque ma faculté de connaître se limite à l'expérience possible et qu'il n'y a pas d'expérience de la chose en soi (nous n'expérimentons que des phénomènes plus ou moins matériels).

Le postkantisme allemand n'a retenu de Kant que la dimension idéaliste de son œuvre (en bannissant la chose en soi : par exemple l'identité de la substance et du sujet chez Hegel), limitant le monde à ce qui en est accessible aux êtres humains, voire en affirmant qu'il n'y a pas d'autre monde que celui accessible aux êtres humains. Chez Kant lui-même, on doit bien admettre que, plus que reconnues comme des réalités effectives, les choses en soi ne servent qu'à rappeler à l'homme le destin de sa finitude. Le corrélationnisme, tel que Quentin Meillassoux le définit, est précisément la primauté exclusive accordée à la relation entre la pensée humaine et son postulat ${ }^{11}$. Le projet de l'Ontologie orientée vers l'objet - que ce soit chez Graham Harman, Timothy Morton, Ian Bogost ou Levi R. Bryant - sera de proposer une approche anticorrélationniste des objets, c'est-à-dire - et spécialement pour ce

\footnotetext{
${ }^{9}$ Emmanuel Kant, Critique de la raison pure [1781], Euvres philosophiques, tome I, édité sous la direction de Ferdinand Alquié, Paris, Gallimard, coll. « Bibliothèque de la Pléiade », 1980, p. 804.

${ }^{10}$ Ibid., p. 863.

${ }^{11}$ Cf. Quentin Meillassoux, Après la finitude. Essai sur la nécessité de la contingence, Paris, Le Seuil, coll. « L’Ordre philosophique », 2006.
} 
que j'en ferai par la suite avec les images - non limitée aux capacités spatiotemporelles de représentation de la conscience humaine.

Un siècle après Kant, parallèlement à ce qui se passe dans d'autres domaines du savoir où la question de l'expérience et de la spatiotemporalité traditionnelle des objets est remise en cause (impressionnisme, relativité restreinte, mécanique quantique, etc.), deux philosophes vont ouvrir une brèche à l'intérieur du corrélationnisme et des limites de ce qui du monde extérieur est seulement accessible aux êtres humains. Ils vont nous mettre sur la voie de ce que l'on ne saurait se débarrasser à si bon compte de ce qui de l'objet échappe à notre saisie, et que notre incapacité à atteindre à l'inconnaissable de l'objet est en tant que telle une composante intraitable de la relation que nous entretenons avec lui, avec ses fronts connaissables, comme le recto est indissociable du verso. Si l'on pouvait couper une feuille de papier en deux pour en détacher le verso, on ne ferait que le remplacer par un autre verso : il ne peut pas y avoir de recto sans verso. Nouveauté : contrairement à ce qu'affirmait Kant et les postkantiens, je perçois toujours l'objet accompagné $d u$ non-accès à la part qui m'en reste impénétrable.

Edmund Husserl, pour commencer par lui, est présenté par les nouveaux philosophes de l'objet comme un «idéaliste centré sur l'objet ${ }^{12} »$ : d'un côté, l'intentionnalité ancre la phénoménologie dans le corrélationnisme et dans ce qui de la chose est seulement abordable par la conscience humaine mais, d'un autre, la phénoménologie a introduit un "parfum de réalisme ${ }^{13}$ » dans la philosophie idéaliste (le retour promis aux choses-mêmes). "Quoique Husserl sans tienne au seul domaine de l'intentionnalité, il a montré qu'une faille remarquable traversait ce domaine : un abîme entre les objets et leurs qualités ${ }^{14}$. »

Husserl hérite le concept d'intentionnalité de Franz Brentano, qui avait proposé une relecture de la vieille intentionnalité médiévale. Contrairement à ce qu'avait avancé Descartes dans le moment de la découverte solipsiste du cogito, Brentano avait attiré l'attention sur le fait que la conscience n'est jamais vide d'objet ${ }^{15}$. L'acte conscientiel est toujours une visée: pas de cogito sans un cogitatum, pas de «Je pense » qui ne soit pas en même temps un «Je pense quelque chose ». Mais l'objet brentanien n'existait pas en dehors de la conscience. En commençant par mettre le monde naturel entre parenthèses et en excluant qu'il puisse exister des objets que la conscience ne pourrait pas observer, Husserl semble reprendre à son compte le programme de son mentor. En réalité, ce penchant idéaliste de Husserl est « compensé par son admirable capacité à traiter des perceptions comme de réalités authentiques ${ }^{16} »$. S'il n'y a pas de monde à l'extérieur de la conscience, Husserl va reprendre le projet de Kasimierz Twardowski d'ajouter à la psychologie brentanienne la référence à un monde extérieur pour l'injecter à l'intérieur de la sphère immanente de la conscience.

Dans L'Objet quadruple, Harman souligne deux lignes de tension vers l'objet dans la philosophie husserlienne de l'objet intentionnel : la théorie de l'esquisse et la question des traits éidétiques. Cette seconde est, à mes yeux, moins husserlienne que l'effet de la «correction» de Husserl par Harman, ce dont Harman convient volontiers ${ }^{17}$ : je choisirai donc de présenter uniquement la première et, pour

\footnotetext{
${ }^{12}$ Graham Harman, L'Objet quadruple. Une métaphysique des choses après Heidegger, traduit de l'anglais (États-Unis) par Olivier Dubouclez, Paris, PUF, coll. « MétaphysiqueS », 2010, p. 27.

${ }^{13}$ Ibidem.

${ }^{14}$ Ibid., p. 27-28.

${ }^{15}$ Franz Brentano, La Psychologie au point de vue empirique [1873], traduit de l'allemand par Maurice de Gandillac, Paris, Aubier-Montaigne, coll. « Philosophie de l'esprit », 1944.

${ }^{16}$ Harman, L'Objet quadruple, op. cit., p. 30.

${ }^{17}$ Ibid., p. 35-36. Correction heideggerienne.
} 
m'éloigner délibérément de l'infléchissement de L'Objet quadruple, je vais la présenter autrement. Quoique tout objet se donne entièrement à la conscience à chaque instant, par exemple à voir, nous n'en percevons successivement que des « esquisses [Abschattungen] ». La perception de toutes les faces de n'importe quel objet s'étale dans le temps, comme lorsque nous tournons autour d'une statue. Aucun instant ne permet de percevoir la totalité de l'objet présent (on n'atteint pas non plus cette totalité en additionnant les faces) : ainsi y a-t-il toujours une autre face de l'objet, qui me reste à jamais dérobée en tant qu'autre face. Harman parle ailleurs d'un " retrait horizontal ${ }^{18}$ » de l'objet. Que l'objet tourne ne supprime pas l'autre face, mais la déplace ${ }^{19}$. L'objet ne coüncide jamais avec ma perception actuelle. L'unité de l'objet intentionnel est garantie chez Husserl par une intuition intellectuelle d'ailleurs mystérieuse.

Martin Heidegger, pour sa part, va déplacer la question husserlienne idéaliste de l'objet intentionnel (sensuel) en rapport avec ses qualités sensuelles, vers l'objet réel et ses relations à ses qualités sensuelles. Ici, le retrait de l'objet sera un "retrait vertical $^{20} \gg$ plus radical. C'est ce que l'on rencontre dans la célèbre analyse de l'outil de $\hat{E}$ tre et Temps $^{21}$, qui avait déjà été mise en place dans les cours de Fribourg de 1919. Celle-ci repose sur la distinction entre, d'une part, "l'être-à-portée-de-lamain [Zuhandenheit] » et, d'autre part, "l'être-sous-la-main [Vorhandenheit] ${ }^{22}$ ». " L'être-à-portée-de-la-main » correspond à l'objet comme outil, pris dans un réseau d'usages (l'outil n'existe pas comme quelque chose d'isolé), comme maniabilité, disponibilité ; «l'être-sous-la-main » désigne la présence de l'objet en elle-même subsistante. Dans le monde naturel, l'objet existe d'abord pour lui-même et ensuite il devient outil pour (zu) l'être humain (Dasein), qui de par la manière dont il lui apparait y voit la possibilité de tel ou tel recours ${ }^{23}$. En revanche, pour l'homme, qui est toujours déjà jeté dans un contexte tenu pour acquis, la première encontre avec les objets est d'abord celle de l'employabilité pour sa préoccupation. Ce n'est qu'ensuite que la question de la présence authentique de l'être-substrat de l'objet peut se poser comme « séquestrée ${ }^{24}$ » par l'ustensilité. Dans la mesure où c'est l'apparaître de l'objet qui dirige son employabilité comme outil, l'objet ne se confond jamais avec son apparence (son usage). Même l'outil est à peine remarqué, sinon - précise Heidegger - quand il tombe en panne. «La réalité glisse hors de ma vue dans un monde infernal perpétuellement voilée ${ }^{25}$. »

Husserl et Heidegger ont replacé, avec d'autres (Whitehead, Souriau, Dagognet, Michel Serres, Bruno Latour), l'objet au cœur de la philosophie - ce qui s'était perdu

\footnotetext{
${ }^{18}$ Graham Harman, Weird Realism : Lovecraft and Philosophy, Winchester/Washington, Zero Books, 2012, p. 31.

${ }^{19}$ Edmund Husserl, Idées directrices pour une phénoménologie [1913], traduit de l'allemand par Paul Ricoeur, Paris, Gallimard, coll. « Tel», 1985, p. 135-138.

${ }^{20}$ Harman, Weird Realism : Lovecraft and Philosophy, op. cit., p. 31.

${ }^{21}$ Martin Heidegger, Etre et Temps [1929], traduit de l'allemand par Emmanuel Martineau, Paris, Authentica, 1985, p. 70-74.

22 Je profite de la présente occasion pour corriger une distraction regrettable dans la première édition de L'Étrange Créature du lac noir de Jack Arnold. Aubades pour une zoologie des images, op. cit., p. 163 : il faut inverser Zuhandenheit et Vorhandenheit dans les parenthèses. Pour le reste, le sens est intact.

${ }^{23}$ Je suis ici la leçon de la «pré-sence » de Françoise Dastur : Heidegger et la question du temps, Paris, PUF, coll. « Philosophies », 2015, p. 79.

${ }^{24}$ Harman, L'Objet quadruple, op. cit., p. 127.

${ }^{25}$ Ibid., p. 47. La lecture que fait Harman de Heidegger s'oppose à l'interprétation pragmatiste dominante du philosophe allemand aux États-Unis (Hubert Dreyfus, Mark Okrent). La déformation de l'objet est à la fois pratique et théorique : on n'épuise pas l'être du marteau en l'utilisant, mais pas non plus en le regardant.
} 
depuis Aristote -, sans pour autant sortir du corrélationnisme, et ont mis fin à des siècles de démolition ou d'ensevelissement des objets ${ }^{26}$.

\section{ICONOLOGIE ORIENTEE VERS L'IMAGE}

Le pari de l'Ontologie orientée vers l'objet est que l'on peut parler malgré tout des objets en tant que certaines de leurs parties dépassent irréductiblement nos puissances représentatives et cognitives. En un sens, il n'y pas d'autre ontologie possible puisque ce qui se retire de l'objet ne peut être dissocié de ce que j'appréhende de l'iceberg de l'objet. D'ailleurs, est-il si exact de dire que l'on ne peut rien du tout de cette part inconnaissable? Il ne faudrait alors plus différencier penser et connaître, mais désormais connaître et connaître. C'est la solution proposée par Graham Harman : ce n'est pas parce que nous ne pouvons pas connaître ce qui se retire toujours des objets que nous ne pouvons pas connaître quelque chose au sujet de ce retrait par le biais de ce qui nous en apparaît. La nuance est décisive.

\section{1.}

La perspective relationniste qui est la mienne sur les images - que j'ai rappelée en commençant mais sur laquelle je ne peux revenir ici en détail ${ }^{27}-$ m'amène à affirmer qu'il faut en même temps toujours envisager les images en tant que ce que l'on peut penser des images, c'est-à-dire des relations qu'elles entretiennent avec et dans leur environnement iconique, ne se réduit pas à la relation entre elles et les hommes ni ce qui des autres relations est connaissable par l'homme. Ce point va très vite poser de sérieuses difficultés tant rien ne semble plus opposer aux démarches des ontologues de l'objet que les thèses pour lesquelles l'objet n'existe que par ses relations. Il y aurait même là au premier abord deux visions des objets irréconciliables.

Évidemment faut-il, là encore, s'entendre sur le sens de ma proposition : nous sommes des hommes, nous y sommes voués, et c'est encore en tant qu'hommes que nous pouvons dire que nous ne devons pas limiter notre pensée des images à ce que nous sommes capables d'en connaître en tant qu'hommes. Certes, il est aisé de montrer qu'il y a une incompatibilité à parler en tant qu'êtres humains de ce qui échappe à l'accès humain, et de pointer que penser qu'il y a de l'impensable revient ipso facto à le penser comme impensable, donc à l'annuler en tant qu'impensable. Autre manière de tourner l'objection : il est impossible pour un être humain de se représenter un monde sans êtres humains sans s'y portraire comme celui qui prend acte d'un monde sans l'être humain. La première formulation reproche au penseur de prétendre regarder ici si ce qui ne s'y trouve pas s'y trouve quand même ; la seconde, d'aller voir ailleurs qu'il y est.

Cependant, y a-t-il autre chose ici qu'un cercle vicieux purement logique (noncontradiction : rien ne peut être à la fois présent et absent, pensé et impensé) ? Je ne cherche d'ailleurs pas à le nier. Mais cette objection ne porte que sur les prémisses. Or la validité d'un système de pensée, quand bien même il comporte des erreurs logiques - et quel système n'en a pas ? -, tient bien plutôt dans ses résultats. Whitehead : « La vérification d'un schème rationaliste doit être cherchée dans son succès général, et

\footnotetext{
${ }^{26}$ Sur ces deux termes, $c f$. ibid., p. 14-20.

${ }^{27}$ Je renvoie de nouveau à la seconde partie de mon ouvrage Cinéma et cristaux. Traité d'éconologie, $o p$. cit., et notamment à son chapitre « Écologie».
} 
non pas dans la certitude particulière ou dans la clarté initiale de ses premiers principes ${ }^{28}$. 》 En limitant l'immensité du temps et de l'espace à la seule région d'adéquation humaine, il faut bien reconnaître que l'on n'a pas gagné grand-chose, voire on y a même beaucoup perdu. Je viens de montrer comment le programme anticorrélationniste de l'ontologie orientée vers l'objet, auquel on peut faire le même type d'objection ${ }^{29}$, permet de moduler ce type d'affirmation. Il revient aussi à l'homme d'affronter la part inhumaine des images.

Malgré des apparences trop rapides, souvent reprochées aux ontologies des objets, malgré l'antirelationnisme des ontologues de l'objet pour qui les relations entre objets ne se situent qu'à un niveau sensuel, tandis que l'objet réel demeure dans une radicale quarantaine, il n'y a pas d'incompatibilité de principe entre le relationnisme des images et l'anticorrélationnisme éconologique. En effet, toute ontologie de l'objet n'a pas une texture heideggerienne (antirelationniste) du genre de celle de Harman. Chez Harman, les théories relationnistes des objets sont contraires aux perspectives anticorrélationnistes de l'Ontologie orientée vers l'objet. Le relationnisme, pour lequel n'y a pas d'être sans relation, non seulement restreint l'objet à son « être-àportée-de-la-main » et à son apparence sensuelle (l'usage dépend d'un contexte : un même couteau peut servir à cuisiner ou à tuer) et appartient aux stratégies d'éviction des objets en ce qu'il les « ensevelit» sous les relations au détriment de leur "réalité intrinsèque $^{30}$ », mais il s'étend bien au-delà de la seule sphère d'appartenance humaine: "S'il n'existe aucun moyen de faire qu'un marteau soit parfaitement présent à ma pensée ou à mon action, il n'existe aucun moyen non plus de faire que le coton soit présent pour le feu ou le verre présent pour les gouttes de pluie ${ }^{31}$. » Il y a une « inadéquation permanente de toute relation en tant que telle ${ }^{32}$ ».

Je défends la thèse contraire : en général, et en matières d'images en particulier, le relationnisme est compatible avec l'anticorrélationnisme des philosophie orientée vers l'objet ${ }^{33}$. Et ce, pour deux raisons. Tout d'abord, même chez Harman, la " réalité intrinsèque » de l'objet apparaît souvent comme une relation de l'objet à lui-même, comme lorsqu'il écrit que «l'objet lui-même ne possède pas ses propres caractéristiques essentielles ${ }^{34} »$. Et qu'est la propriété si ce n'est une relation ? Et, au fond, qu'est-ce que l'absence de relation si ce n'est une relation?

La seconde raison est plus profonde : la relation n'est pas la corrélation. Dans la corrélation, l'un des deux termes ne peut pas être pensé sans cet autre-ci. Au contraire, le relationnisme, en affirmant que tout être n'est que parce qu'il est en relation, en posant qu'aucun terme ne peut être pensé sans le moindre autre quel qu'il soit, permet aussi de penser qu'il puisse toujours exister d'autres relations pour un terme que celle

\footnotetext{
${ }^{28}$ Alfred North Whitehead, Procès et réalité. Essai de cosmologie [1929], traduit de l'anglais (Angleterre) par Daniel Charles, Maurice Élie, Michel Fuchs, Jean-Luc Gautero, Dominique Janicaud, Robert Sasso et Arnaud Villani, Paris, Gallimard, coll. « Bibliothèque de Philosophie », 1995, p. 53.

${ }^{29}$ Harman, L'Objet quadruple, op. cit., p. 71-79.

${ }^{30}$ Ibid., p. 19.

${ }^{31}$ Ibid., p. 54. Pour Harman, chez Heidegger déjà l'être-sous-la-main ne peut exister qu'en relation (p. 62-64).

32 Ibidem.

33 Whitehead, à qui l'on doit l'un des développements les plus remarquables des philosophies relationnistes, partage avec les tenants de l'Ontologie orientée vers l'objet le même reproche adressé à la philosophie kantienne (ou postkantienne) d'avoir réduit la pensée à l'expérience purement subjective. Je suis longuement revenu sur ce point pour les seconds. Pour le premier, $c f$. Alfred North Whitehead, Procès et réalité. Essai de cosmologie [1929], traduit de l'anglais (Angleterre) par Daniel Charles, Maurice Élie, Michel Fuchs, Jean-Luc Gautero, Dominique Janicaud, Robert Sasso et Arnaud Villani, Paris, Gallimard, coll. « Bibliothèque de Philosophie », 1995, p. 40.

${ }^{34}$ Harman, L'Objet quadruple, op. cit., p. 119.
} 
qu'il a avec un autre terme, et que donc rien d'aussi clos et monodirectionnel que la corrélation, notamment la corrélation hommes-objets, ne saurait imposer ses lois. On peut nombrer d'autres relations, entre objets, entre images, sans passer par la médiation constitutive de l'homme, image et fiction parmi les autres, comme les autres $^{35}$.

2.

Le modèle éconologique, pour lequel les relations constitutives des objets (des images) échappent à l'accès humain, trouve son origine chez Aby Warburg. À l'instar de Kant, Warburg a ouvert la voie d'une vie des images et des figures indépendantes des hommes (intentions des artistes, exigences des commanditaires, etc.), par les migrations des «formules de pathos [Pathosformeln]»d'images en images, d'époques en époques, selon des cartographies spatiotemporelles dont l'originalité fondatrice et distinctive de toute forme d'héritage (typiquement pensé sur le modèle de la juridiction humaine) est d'être proprement inhumaines, par spectres, échos, enfouissements, mutations, et ainsi de suite. Wölfflin, l'antipode de Warburg, avait également appelé de ses vœux en 1920 une «histoire de l'art sans noms propres [Kunstgeschichte ohne Namen] ${ }^{36}$ ", c'est-à-dire tout le contraire de l'histoire de l'art héroïque et crypto-romantique qui mettait alors en avant le génie individuel des artistes. Robert Klein avait fait remarquer que la discipline inventée par Warburg n'avait elle-même pas de nom ${ }^{37}$ : du moins dans une langue humaine.

Cette conviction anime l'entièreté de l'œuvre intellectuelle de Warburg, avec plus ou moins d'intensité, depuis les premiers textes (sur Botticelli ou le mariage archiducal de 1589) jusques aux friches de l'atlas terminal. Le geste théorique warburgien est, justement, co-constitutivement anticorrélationniste et relationniste : il existe une symbiose des images que l'homme ne peut espérer approcher qu'indirectement par l'écume de ses déformations et de ses variations phénoménales mais qui en tant que telle lui reste à jamais interdite (anticorrélationnisme); cette vie n'est composée que de relations environnementales et développementales entre les images (relationnisme). L'inhumanité irréductible des images, c'est-à-dire l'inaccès à jamais répété de l'homme aux processus foncièrement imageants, la nature benthique de la survivance, se traduit parfaitement dans la tension polaire des symboles visuels entre astrum et monstrum. Tous les faits de culture présentent une inquiétante dualité. Pour l'exprimer, Warburg détourne la locution latine «par des sentiers ardus jusqu'aux étoiles [per aspera ad astra] », qui relève précisément de la question de l'accès humain, en "per monstra ad astra », des monstres aux étoiles : soit deux régimes radicaux d'inhumanité. La dialectique du monstre et de l'astre, du proche et du lointain, du dynamisme maximal et du relâchement, de l'archaïque et $d u$

\footnotetext{
${ }^{35}$ Cela ne voudra évidemment pas dire que toutes les images sont égales et que l'homme vaudrait autant qu'une carte postale. Mais que toutes les images, quelles qu'elles soient, le sont à titre égal. L'éconologie, à la suite d'Aby Warburg pour qui toutes les images devaient être traitées comme des documents de la culture d'égale dignité, est une iconologie plate pour laquelle toutes les images - et l'homme en est une pour de multiples raisons et sous des acceptions diverses - sont sur un pied d'égalité ontologique.

${ }^{36}$ Heinrich Wöllflin, Réflexions sur l'histoire de l'art, «Pro domo. Justification de mes "Principes fondamentaux de l'art" » [1920], traduit de l'allemand par Rainer Rochlitz, Paris, Flammarion, coll. « Champs », 1997, p. 43.

37 Robert Klein, La Forme et l'intelligible, Paris, Gallimard, coll. «Bibliothèque des Sciences humaines », 1970, p. 224.
} 
linguistique, du mythe et de la raison, du chaos et de l'ordre, du refoulement et du symptôme, comme soubassement d'une théorie sociologique de l'énergie, culmine dans l'analyse des symboles et l'intérêt warburgien pour l'astrologie (les planètes sont bienfaisantes ou maléfiques selon les contextes: démons décidant de l'existence humaine ou repères pour l'orientation), le Picatrix médiéval de la tradition arabe, ou encore Le Pourceau monstrueux de Landser à huit pattes d'Albrecht Dürer (1496), inspiré par Sebastian Brant, et les caricatures zoomorphiques des xylographies anticatholiques $\mathrm{du} \mathrm{XVII}^{\mathrm{e}}$ siècle allemand. Cette thématique s'est particulièrement développée chez le dernier Warburg, après l'internement psychiatrique - l'Atlas Mnémosyne regorge d'images de monstres -, à travers le motif du «combat avec le monstre [Kampf mit dem Monstrum $]^{38}$ » au cœur de «la reconstitution des formes pures des dieux classiques à la Renaissance ${ }^{39} »-1$ 'homme de la Renaissance devant prendre position dans la lutte entre des forces contradictoires - ; mais également tapi en lui-même, pour sa propre renaissance psychologique, et dont la théorie freudienne de l'inconscient donnait depuis quelques années une idée à jamais approximative de l'inaccessibilité en tant que telle (ou l'inconscient deviendrait conscient...). « La fonction mnémique [sic] est un combat à "double tendance" pour occuper l'espace de pensée $^{40}$. » Toute image, d'une manière générale, est un état d'équilibre entre deux pôles opposés dont le «combat avec le monstre » métaphorise les tensions internes.

Si d'un côté, la réinvention de la tradition ne peut pas être absolument déconnectée de l'implication humaine, il n'en reste pas moins que se jouent dans la survivance de l'Antique à partir du Quattrocento (pour m'en tenir à cette période clé des études warburgiennes) des mouvements et des enjeux engageant d'autres strates spatiotemporelles que celles des existences humaines. La vie des images est la chose en soi de l'iconographie. En revanche, les successeurs de Warburg se sont empressés de «recorrélationniser» sa pensée des images et de rabattre, comme je l'ai déjà rappelé, la survivance, avec sa part d'inconscient, d'abîme, d'énigme et de terreur, sur la notion bien domestiquée et précisément pensée par le tribunal humain de l'héritage. Nous tenons là un nouveau chapitre venant s'ajouter à la longue histoire du « rituel théorique d'exorcisme ", sur lequel Didi-Huberman est précisément revenu, chez Gombrich, Saxl ou Panofsky à l'égard de leur mentor, et qu'il n'est nul besoin de redire ici (repli sur l'influence, privilège de l'héritage sur la survivance, etc.). À bien des égards, ils auront été les Fichte et les Hegel de Warburg. Le néokantisme initial de Panofsky, par exemple, l'a amené à réduire les thèses warburgiennes à une théorie de la connaissance dans laquelle on ne peut pas voir une image sans la lire et pour laquelle un spectateur illettré ne saurait pas comprendre le moindre tableau ${ }^{41}$. Pour comprendre, il faut "posséder certaines connaissances littéraires, antérieures à l'analyse que nous faisons ${ }^{42}{ }$. Et si certaines remarques de Panofsky au sujet du stade

\footnotetext{
${ }^{38}$ Aby Warburg, «Idées générales » [1927], cité in Ernst Gombrich, Aby Warburg. Une biographie intellectuelle, , suivi d'une étude de Fritz Saxl, traduit de l'anglais (Angleterre) par Lucien d'Azay, Paris, Klincksieck, 2015, p. 238.

${ }^{39}$ Gombrich, Aby Warburg. Une biographie intellectuelle, op. cit., p. 190.

${ }^{40}$ Warburg, « Notions élémentaires », cité in Maud Hagelstein, Origine et survivances des symboles. Warburg, Cassirer, Panofsky, Hildesheim/Munich/New York, Georg Olms, coll. « Europea Memoria », 2014, p. 72.

${ }^{41}$ Erwin Panofsky, Essais d'iconologie. Les thèmes humanistes dans l'art de la Renaissance [1939], traduit de l'anglais (Allemagne) par Claude Herbette et Bernard Teyssèdre, Paris, Gallimard, coll. « Bibliothèque des Sciences humaines », 1967, p. 26 : «L'aborigène des brousses australiennes [...] serait incapable de reconnaître le sujet d'une Cène ; elle ne saurait lui suggérer d'autre idée que celle d'un repas dans une ambiance tendue. »

42 Erwin Panofsky, La Pespective comme forme symbolique, "Contribution au problème de la description d'œuvres appartenant aux arts plastiques et à celui de l'interprétation de leur contenu »
} 
proprement iconologique de l'analyse (le troisième et dernier, après les stades préiconographique et iconographique), portant sur « l'essence ${ }^{43}$ » des œuvres dans leur singularité, indiquent néanmoins un dépassement - jamais complètement réalisé (le tout s'emboîte plutôt comme des poupées russes) - de la connaissance vers la pensée, il s'agit d'une pensée toujours étalonnée sur l'échelle des potentialités humaine ${ }^{44}$. Ernst Gombrich, quant à lui, est allé encore plus loin en " assimil[ant] les œuvres d'art à des "wrappings of verbal statements", ce qui revient à peu près à dire qu'elles sont comme "l'emballage d'énoncés verbaux" 45 ", et en identifiant l'iconologie à une linguistique de l'image ${ }^{46}$. Au passage, sans nier la vérité partielle de ces méthodes, a été ruinée l'activité chthonienne des images dans leur continent souterrain. Contre Warburg, Gombrich et Panofsky ont mis en place une iconologie orientée vers l'esprit humain. L'éconologie propose de revenir à l'intuition majeure de Warburg - celle d'une iconologie décérébrée ou acéphale - et d'en tirer les pleines conséquences : non seulement celle de la vie des images, comme on l'a vu précédemment, mais également de ce qui s'y tient plus radicalement en germe et que je propose de nommer une iconologie orientée vers l'image.

\section{3.}

Dans l'éconologie, les images ne sont pas à jamais isolées les unes des autres dans leur être mais sont des rapports écologiques entretenant des relations non ouvertes à l'accès humain : si nous ne pouvons pas les connaître au même titre que ce qui nous apparaît des images, nous pouvons néanmoins en parler. Ceci suppose que pour échapper à l'apparente contradiction de l'anticorrélationnisme et du relationnisme, l'image éconologique ne soit pas une image-chose mais une image-processus, à savoir l'ensemble mutualiste composé par l'image et son environnement iconique (autres images, théories philosophiques, etc.). Les relations entre ce que l'on appelle traditionnellement les images ne sont jamais des relations externes mais des relations internes. S'ils s'opposent à l'idée que les objets réels entretiennent entre eux des relations, les ontologues de l'objet soutiennent fréquemment, en revanche, qu'il existe des relations à l'intérieur des objets. Tout en affirmant que « les relations ne peuvent pas être ontologiquement internes à leurs termes ou aux objets qu'elles relatent » et que « les objets ne sont pas constitués par leur relations au reste du monde ${ }^{47}$ », Levi R.

[1931], traduit de l'allemand sous la direction de Guy Ballangé, avec une préface de Marisa Dalai Emiliani, Paris, Minuit, coll. « Le Sens commun », 1976, p. 239.

${ }^{43}$ Ibid., p. 16.

${ }^{44}$ Ibid., p. 28 : « Pour nous saisir de ces principes, nous avons besoin d'une faculté mentale comparable à celle du diagnosticien - faculté que je ne saurais mieux définir que par le terme plutôt discrédité d'“intuition synthétique", et qui peut s'être mieux épanouie chez un profane, s'il est doué, que chez un docte érudit. » Que recoupe exactement ce « don »? Panosfky ne précise pas.

${ }^{45}$ Otto Pächt, Question de méthode en histoire de l'art [1977], avec une introduction de Delphine Galloy, traduit de l'allemand par Jean Lacoste, Paris, Macula, coll. « La Littérature artistique », 2017, p. 40.

${ }^{46}$ Cf. Ernst Gombrich, L'Art et l'illusion. Psychologie de la réprésentation picturale [1959], traduit de l'anglais (Angleterre) par Guy Durand, Paris, Gallimard, coll. « Bibliothèque des Sciences humaines », 1971. À la fin de sa vie, Gombrich a remis en cause le modèle conventionnaliste, qu'il avait largement contribué à forger, dans «Image and Code: Scope and Limits of Conventionalism in Pictorial Representation », in Wendy Steiner (dir.), Image and Code, Ann Arbor Horace H. Rackham School of Graduate Studies/Universty of Michigan, 1981.

${ }^{47}$ Levi R. Bryant, The Democracy of Objects, Londres, Open Humanities Press, coll. « New Metaphysics », 2011, p. 68. 
Bryant distingue entre les «exorelations » et les «endorelations »: ces dernières « constituent la structure interne des objets indépendants des autres objets ${ }^{48}$ ».

Comme n'importe quel objet, l'image n'est pas un quoi mains un quand. Il y a image quand les relations externes d'une image avec d'autres images ou d'autres entités de son environnement iconique (objets, discours, êtres vivants, etc.) atteignent une clôture opérationnelle telle que leur composition devient capable de perturbations de rencontre en termes d'informations sur une consistance interne. Comme le note l'écologue et primatologue japonais Kinji Imanishi, après Jakob von Uexküll ou Georges Canguilhem : "L'être vivant et son environnement ne forment qu'un, et cela même représente le véritable être vivant ${ }^{49}$. " Être en vie ne se réduit pas à avoir un organisme $^{50}$. Traduisons : l'image (qui est une forme de vie) et son environnement ne forment qu'un, et c'est cela même qui est l'image véritable. Chaque image n'est rien d'autre qu'un entrelacs nodal, étoilé et atemporel de relations à d'autres images, actuelles ou virtuelles, présentes ou absentes, passées, présentes ou futures, proches ou lointaines, et ainsi de suite, mais également à tout ce qui n'est pas une image mais intervient, à des degrés et des titres divers, dans son environnement iconique. Ces relations ne sont ni externes à l'image ni accidentelles : elles constituent le seul être stratifié et nébulaire de l'image. Je propose de nommer l'image ainsi entendue images - avec la marque permanente du pluriel même au singulier pour en conserver à l'esprit la composition interrelationnelle. C'est cette images dont l'existence n'est que partiellement actualisée dans l'image que je vois. Parce qu'elle est toujours une images, dont la plus grande partie est au-delà des capacités spatiotemporelles de la perception humaine, l'image n'est jamais la somme de ses qualités sensibles, à quoi l'empirisme et le constructivisme perceptif réduisent tout l'être de tout objet. Ces qualités phénoménales ne sont que la dimension des processus imageants intersectant avec notre plan d'existence humain. Elles ne sont pas plus l'images que le dérèglement météorologique local qui m'accable n'est le réchauffement climatique.

\section{4.}

Trop souvent, l'iconologie se limite aux images observables et à ce qui de leurs relations est intelligible par l'homme. Comme la philosophie, l'iconologie a longtemps été restreinte à la corrélation entre les hommes et les images, au sens où il parait aller de soi qu'une image est perçue: que serait un tableau non vu, par exemple ? Ce type d'affirmations, qui semble tomber sous le bon sens, a en réalité été dramatique pour l'iconologie. Il passe, par exemple, tout à fait à côté d'un point que Diderot avait soulevé s'agissant de la peinture, et que Michael Fried a particulièrement mis en avant dans son étude sur l'invention du spectateur moderne par l' "absorbement» de la peinture dramatique (chassant le spectateur de devant la toile) ou de la peinture pastorale (invitant celui-ci à l'habiter) - dans les décennies ayant précédé la Révolution française : un tableau n'est pas nécessairement fait pour

\footnotetext{
${ }^{48}$ Ibidem.

${ }^{49}$ Kinji Imanishi, Le Monde des êtres vivants. Une théorie écologique de l'évolution, avec une préface de Pamela Asquith et une postface de Baptiste Lanaspèze, traduit du japonais par Anne-Yvonne Gouzard, Marseille, Wildproject, coll. « Domaine sauvage », 2011, p. 82.

${ }^{50} \mathrm{Il}$ existe, en outre, des vies biologiques non organiques, comme la vie des cellules par exemple. Pour plus de détails sur la vie non organique des images, cf. mon ouvrage Cristaux et cinéma Traité d'éconologie, op. cit., le chapitre « Biologie ».
} 
être $v^{51}$, c'est-à-dire ne doit pas nécessairement être regardé en tant qu'il est fait pour être vu.

Je reviens sur la comparaison avec le réchauffement climatique. Le réchauffement climatique en tant que tel est insituable, global, inaccessible à la représentation humaine. Nous n'en percevons que des manifestations ponctuelles parfois divergentes : augmentation de la température ici, baisse ailleurs, multiplication des catastrophes naturelles, fonte du pergélisol, régression des glaciers, acidification des océans, déplacement des aires de répartition de certaines espèces animales, et ainsi de suite. Le réchauffement climatique, quant à lui, implique des entités qui existent bien au-delà de l'atmosphère terrestre et sa majeure partie concerne les temps à venir sur plusieurs siècles. Le philosophe américain Timothy Morton a proposé d'appeler « hyperobjets » cette manière d'objets, transcendant bien souvent la distinction entre la nature et la culture, qui échappent aux capacités humaines de représentation, d'imagination ou de cognition : pollution, radiations nucléaires, trous noirs, boson de Higgs, masse des déchets plastiques, machinerie du capitalisme, etc.

Dans Hyperobjects. Philosophy and Ecology after the End of the World, Morton en cinq caractéristiques essentielles de 1'hyperobjet ${ }^{52}: 1 / 1$ 'hyperobjet colle à l'être humain et remet en cause l'existence d'un ailleurs (viscosité), 2/ aucune des manifestations locales de l'hyperobjet n'est l'hyperobjet lui-même (non-localité), 3/ on ne voit qu'une partie de l'hyperobjet à chaque instant (ondulation temporelle), 4/ tout hyperobjet est non euclidien et existe dans un espace de dimensions supérieures à l'échelle tridimensionnelle de l'équipement physiologique humain (phasage), 5/ les hyperobjets peuvent être détectés dans un espace constitué par les interrelations entre les «propriétés esthétiques des objets [aesthetic properties of objects] ${ }^{53}$ » (interobjectivité). Cette dernière propriété montre également que Morton ne partage pas l'antirelationnisme des autres ontologues de l'objet et met sur la piste de ce qu'une pensée réconciliant relationnisme et anticorrélationnisme doive passer par un questionnement esthétique.

Comme n'importe quel hyperobjet, l'images est une entité supradimensionnelle à laquelle l'être humain n'a accès que partiellement et par fronts d'intersection avec son monde. "Toutes les entités (y compris "moi-même") sont timides et se retirent comme les pieuvres qui jettent une encre dissimulatrice quand elles s'enfoncent dans les ombres ontologiques ${ }^{54}$. » L'images - l'image comme relations de l'image avec son environnement iconique - est un hyperobjet: un «étrange étranger [strange stranger $]^{55}$ », qui est aussi une manière d' " étrange plus étrange », d'étrange à ce point étrange qu'il rend l'idée même d'étrangeté étrangère. Où commence une image ? L'image que je vois - notre monde hypermédiatique, s'il l'accentue, ne l'invente pas : simple différence de degré -, toute image, à quelque époque ou en quelque lieu, n'est que l'une des faces d'un hyperobjet iconique bien plus vaste composé de l'ensemble des relations tissées entre les images connectées d'un même environnement iconique. L'images nous jette au-delà de notre cadre spatiotemporel expérimentable. Pour l'exprimer simplement: ce qui sépare l'image objectale de l'images hyperobjectale est, ceteris paribus, identique à ce qui différencie dire que

\footnotetext{
${ }^{51} \mathrm{Cf}$. Michael Fried, La Place du spectateur. Esthétique et origines de la peinture moderne, traduit de l'anglais (États-Unis) par Claire Brunet, Paris, Gallimard, coll. « NRF Essais », 1990.

52 Timothy Morton, Hyperobjects. Philosophy and Ecology after the End of the World, Minneapolis/Londres, University of Minnesota Press, coll. « Posthumanities », 2013, p. 27-95.

${ }^{53}$ Ibid., p. 1.

${ }^{54}$ Ibid., p. 3-4.

${ }^{55}$ Morton, Hyperobjects, op. cit., p. 6.
} 
l'on respire du dioxygène et dire que la pollution cyanobactérienne apparue à l'Archéen (vers - 2,7 milliards d'années) remplit nos alvéoles pulmonaires. Dans le premier cas, vous en restez à ce qui est centré sur l'homme, à ce qui se passe pour l'homme qui parle; dans l'autre, vous parlez à partir de la pensée d'un temps et d'événements qu'aucun homme ne peut se représenter. Les relations entre les images - constituant l'essentiel de l'images - se tiennent dans des parages du même registre inaccessibles à l'être humain ${ }^{56}$.

Cette part des images à laquelle nous n'avons pas accès, si elle ne peut impliquer aucune expérience esthétique pour elle-même par définition, n'est néanmoins pas absente pour autant de notre expérience esthétique globale de l'image et ne constitue nullement, quoiqu'elle ne puisse jamais être regardée, un déni à l'acte de regard au fondement de l'esthétique moderne (la même modernité ne s'accommode-t-elle pas très bien de l'inconscient freudien, qui est un hyperobjet du même type ?). Tout acte de regard se définit également par ce qui reste hors de la vue - que ce reste soit délaissé par le regard lui-même dans son élaboration de la manière de voir une image (exploration, sélection, interprétation, etc.) ou qu'il soit refusé au regard humain purement et simplement. Le projet d'élaborer un modélisme de la vie cachée des images relève aussi le défi d'une réappropriation active de leurs arcanes par l'esprit, c'est-à-dire précisément par le regard.

La comparaison avec un mollusque marin avait, en quelques sorte, lancé ce texte. Qu'on me permette de le conclure par une autre image des mystères océaniques. Dans son beau livre sur l'étude des cachalots en milieu aquatique (à quelques mètres seulement de profondeur : dans leur habitat naturel, la pression est deux cents fois supérieure à celle atmosphérique), François Sarano note, dès le début, que la première fois que l'on se trouve face à un cachalot sous l'eau, « on ne comprend $\operatorname{pas}^{57} »$ : l'animal s'annonce par des «claquements métalliques » (son sonar écholocalisant), puis surgit le "parallélépipède bulbeux » de l'organe à spermaceti, unique dans tous les règnes zoologiques, et ensuite c'est une "muraille», une "masse creusée de cicatrices et de crevasses ». Combien la description diffère de celle des observateurs touristes ou aussi certains savants - subjugués par la chorégraphie des cétacés remontant à la surface de la mer, depuis le rivage ou tel bateau éloigné, ou de ces badauds grimpant sur leurs cadavres échoués et apprivoisés !

Il faudrait voir les images comme Sarano le cachalot: non comme des spectateurs distants, mais au contact. Alors leur vie monstrueuse nous apparaîtrait exactement comme quelque chose qui dépasse notre compréhension.

\footnotetext{
${ }^{56}$ L'images est un bon exemple de ce que l'éconologie doit au cinéma. Car le film, plus que tout autre image, animé mais composé de photogrammes statiques, pelliculaire mais aussi projeté, matériel et immatériel, ici et là-bas, objet qui n'en est pas vraiment un tout en étant un objet à part entière, est luimême insituable spatio-temporellement ( $c f$. Jean-Michel Durafour, L'Étrange Créature du lac noir de Jack Arnold. Aubades pour une zoologie des images, op. cit., p. 167-170).

${ }^{57}$ François Sarano, Le Retour de Moby Dick. Ou ce que les cachalots nous enseignent sur les océans et les hommes, avec une préface de Jacques Perrin, Arles, Actes Sud, coll. " "Mondes sauvages" pour une nouvelle alliance », 2017, p. 25, pour toutes les citations.
} 